\title{
Nanomedicines for delivery of therapeutic proteins and biopharmaceuticals
}

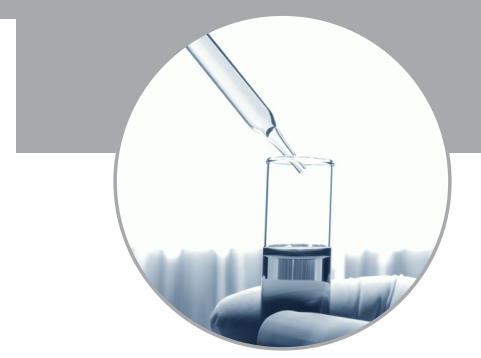

Biopharmaceuticals are a class of therapeutics including recombinant therapeutic proteins, engineered antibodies and genetic material. They are attractive from a medical point of view, but technologically they face major challenges. The delivery of biopharmaceuticals still prevents them from reaching their maximum pharmacodynamic potential, owing to their physicochemical properties, poor stability, permeability and biodistribution. In our research group we have been involved with inter-related projects that intend to optimize strategies to improve the bioavailability of biopharmaceutical drugs after administration. One of the most employed drugs has been insulin, the oldest therapeutic protein, as well as siRNA and gene silencing genetic material. We are interested in formulating such drugs in polymeric nanoparticles, because they encompass an array of advantages compared with classical dosage forms. We also look for tools to correlate in vitro and in vivo absorption rates of biopharmaceuticals, in a rational chronologic sequence of manufacturing parameters, preclinical assessments and requirements for marketing approval.

Bruno Sarmento is a research scientist in the Pharmaceutical Technology Department of the Faculty of Pharmacy of University of Porto, Portugal and a Professor of Pharmaceutics at the Instituto Superior de Ciências da Saúde Norte. His research has focused on delivery of biotechnological drugs by means of colloidal targets, development of new drug delivery systems using polymeric and lipid nanoparticles and therapeutic polymers, and controlling delivery of biopharmaceutical drugs across biological barriers, namely the GI tract, ocular mucosa and blood-brain barrier.

His collaboration with the Pharmaceutical Technology Department began during his doctoral program and was continued afterwards, with the implementation of new methodologies and research projects. He is currently responsible for the supervision of postgraduate masters and doctoral students in the field of nanotechnology and biopharmaceuticals.

The topics of research were a natural selection after the preliminary results within the research group. Then, the laboratory was set up with technologies and equipment to develop and characterize pharmaceutical systems and biopharmaceuticals themselves, giving rise to interesting outcomes in recent years and advances in the understanding of biopharmaceutical formulation and pharmacokinetics.

The group currently maintain collaborating studies with the Faculty of Pharmacy at the University of Copenhagen (Denmark), the Faculty of Pharmacy at the University of Helsinki (Finland), the Faculty of Pharmacy at the University of Pavia (Italy), the Faculty of Pharmacy at the University of Santiago de
Compostela and Barcelona (Spain), the Federal University of Bahia (Brazil) and the Faculty of Pharmacy and Biochemistry at the University of Buenos Aires (Argentina), among others. Partnership is considered essential to enlarge the competences and improve knowledge, as demonstrated by the collaborative outcomes.

\section{Therapeutic proteins \\ \& biopharmaceuticals}

Biopharmaceuticals are complex drug molecules manufactured by biotechnological means, usually involving live organisms or their active components. Biopharmaceuticals are increasingly used in therapy to treat many metabolic and oncologic diseases, to which the advances in the field of pharmaceutical biotechnology were fundamental for these achievements.

Protein drugs represent a significant part of the new pharmaceuticals coming on the market every year and are now widely used to treat or relieve symptoms related to many metabolic and oncologic diseases.

The delivery of therapeutic proteins still prevents them reaching their maximum pharmacodynamic potential, owing to their physicochemical properties, poor stability, permeability and biodistribution. Although the parenteral route is still the primary route of protein administration, research continues on nonparenteral delivery routes. However, the high molecular weight of proteins, combined with their hydrophilic and charged nature, renders transport through membranes very difficult.

For a long time, much of our research activities have focused on the development of new formulations that could provide protein stability,

\section{Bruno Sarmento}

Department of Pharmaceutical Technology Faculty of Pharmacy, University of Porto, Rua Aníbal Cunha, 164 4050-047, Porto, Portugal and Department of Pharmaceutical Sciences, Instituto Superior de Ciências da Saúde - Norte Rua Central de Gandra, I317 4585-|| 6, Gandra, Portugal Tel.: +35। 222078949 Fax: +351 222003977

E-mail: bruno.sarmento@ff.up.pt 


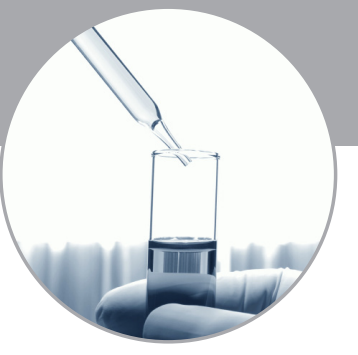

not only during the formulation process but also in storage and delivery. Such formulations are mainly polymeric nanoparticles, whose colloidal nature and surface affinity to mucosa render significant advantagous regarding parenteral and nonparenteral administration.

Another important biopharmaceutical is siRNA therapy, which suppresses different regulatory proteins. siRNA is a process by which a specific mRNA is targeted for degradation as a means of inhibiting the synthesis of the encoded protein. siRNA is an efficient neutral mechanism to protect the genome. The underlying molecular mechanism of gene silencing provides us with short siRNAs, which allow the targeting of any gene with specificity and efficacy. It is now largely preferred over other gene silencing technologies such as antisense oligonucleotides and ribozymes because of its more potent and robust effects in cultured cells and in vivo. However, systemic injection of siRNA presents some limits due to short half-life and high toxicity. In this context, the use of colloidal carriers such as nanoparticles or therapeutic polymers represents an attractive alternative because of their potential to induce controlled release of siRNA in target tissues [1].

\section{Protein nanoencapsulation \& structural analysis}

Most therapeutic proteins are used for lifethreatening and seriously debilitating diseases such as diabetes, cancer, rheumatoid arthritis or hepatitis. The high activity and specificity of proteins compared with the more conventional low-molecular-weight drugs often allows for a better treatment of these diseases. The success of a protein formulation depends not only on the stability of the delivery system but also on the ability of the protein to maintain the native structure and activity during preparation and delivery, as well as during long-term storage of the formulation. However, the production and delivery of these proteins occur under unfavorable stress conditions to both protein stability in the formulation and patients during disease [2]. Denatured or aggregated protein species will not only be therapeutically inactive, but may also cause unpredictable side effects, such as immunogenicity or toxicity. Furthermore, denaturation of genetic material or any other mean of destabilization leads to the loss of its biological activity. Moreover, siRNA molecules are neither trivial nor inexpensive, emphasizing the importance of their stability for use as therapeutics.
Although nanoencapsulation shows promising advantages for protein delivery, little attention has been paid to the effect of nanoencapsulation methodology on protein structure (Figure I) [3]. Different types of forces are responsible for protein physicochemical stability, such as hydrophobic and electrostatic interactions, covalent bonding, hydrogen bonding and van der Waals forces. The complex interaction between these forces places hydrophobic residues toward the interior of the protein while directing hydrophilic residues to the outside, where they interact with the aqueous solvent. Manipulation of proteins can lead to conformational changes, thus exposing hydrophobic areas, resulting in reduced solubility and an increased tendency to aggregate [4]. Also, physical forces related to various processes, such as stirring, pumping, filtration, chromatography and centrifugation, can expose proteins to air-liquid and liquid-solid interfaces resulting in similar undesired effects and loss of structural integrity.

Forms of chemical degradation include deamidation, isomerization, hydrolysis, racemization, oxidation, disulfide formation and $\beta$-elimination. Physical stability is generally defined as the ability of a protein to retain at least its tertiary structure, which is crucial for biological activity. Physical degradation involves reversible or irreversible denaturation through loss of tertiary structure and unfolding with further reactions such us chemical degradation, aggregation and precipitation.

The use of nanoparticles for protein delivery has been a focus in the field of long-term systemic delivery, intended to last for several days or weeks, depending on the degradability and erosion of the nanomeric matrix. We considered it important to examine the structural integrity of therapeutic proteins upon entrapment into different nanoparticle systems $[3,5]$. Owing to their importance to protein physical stability analysis, analytical methodologies are used to examine protein structure and interactions between proteins and polymeric matrices after entrapment. A significant research line of our group is focused on optimizing, characterizing and selecting nanoparticle formulations that show good protein delivery properties, good protein stability and optimal potential in vivo application. Fourier-transform infrared, circular dichroism, microdifferential scanning calorimetry and western blot are examples of techniques used by us to evaluate protein conformation. Moreover, we aim to associate the protein nanoencapsulation 
process to freeze-drying strategies employing cryo- and lyo-protectors to retain and prolong the stability of therapeutic proteins.

\section{Use of in vitro cell models as a tool to correlate the transport of biopharmaceuticals across human intestine}

Since the establishment of our research group we were aware that oral drug delivery is considered the preferred route of administration due to its convenience. However, the transport of drugs, namely proteins and other biopharmaceuticals, across the intestine is a complex and dynamic process that may include the passage across several functional pathways.

The intestinal epithelial layer consists of a cell monolayer, predominantly composed of enterocytes interspersed with mucus-secreting goblet cells, which generally constitutes an effective barrier and prevents the absorption of xenobiotics [6]. Therefore, it is crucial to select an appropriate model for understanding the rate-limiting step in the absorption process.

Some proteins are known to undergo intestinal absorption using specific transporters. The direct uptake of insulin, for example, is thought to occur through binding to specific receptors in enterocytes and rapid internalization to the interstitial space before reaching the blood circulation [7]. Also, strategies have been utilized to increase the paracellular permeability of insulin by junctional modulation [8].

Another approach for protein intestinal absorption involves $\mathrm{M}$-cells that are scattered throughout the gastrointestinal mucosa and have the ability to take up proteins and nanoparticles [9]. We have studied the retention of drugs in their absorptive sites by mucoadhesive carriers and their direct uptake, which we believe may act as a synergic factor to improve the oral bioavailability of therapeutic proteins.

Cell lines have the potential to illustrate much of the complexity involved in oral bioavailability. A monolayer of Caco-2 cells is a well-established in vitro model for predicting the absorption of orally administered compounds in humans, including proteins. However, despite the widespread use of the Caco-2 model, it suffers from significant shortcomings that render it less than optimal as a one-stop cell-based permeability-screening tool. It is a homogenous cell system, whereas the human intestinal epithelium is composed of more than eight defined cell types. So, we propose more elaborate models based on Caco-2 cells, namely the
Caco-2/HT29 co-culture, a mucus-producing goblet cell line [10], well correlated with in vivo data [11] and the Caco-2/Raji B lymphocytes model [12], a reproducible model expressing the M-cell phenotype. Moreover, we are currently optimizing an in vitro cellular model based on Caco-2 cells to partially reproduce the characteristics of intestinal enterocytes, with HT29 cells, mucus-secreting cells and M-cells in order to design a model that more accurately mimics the small intestinal epithelial layer and

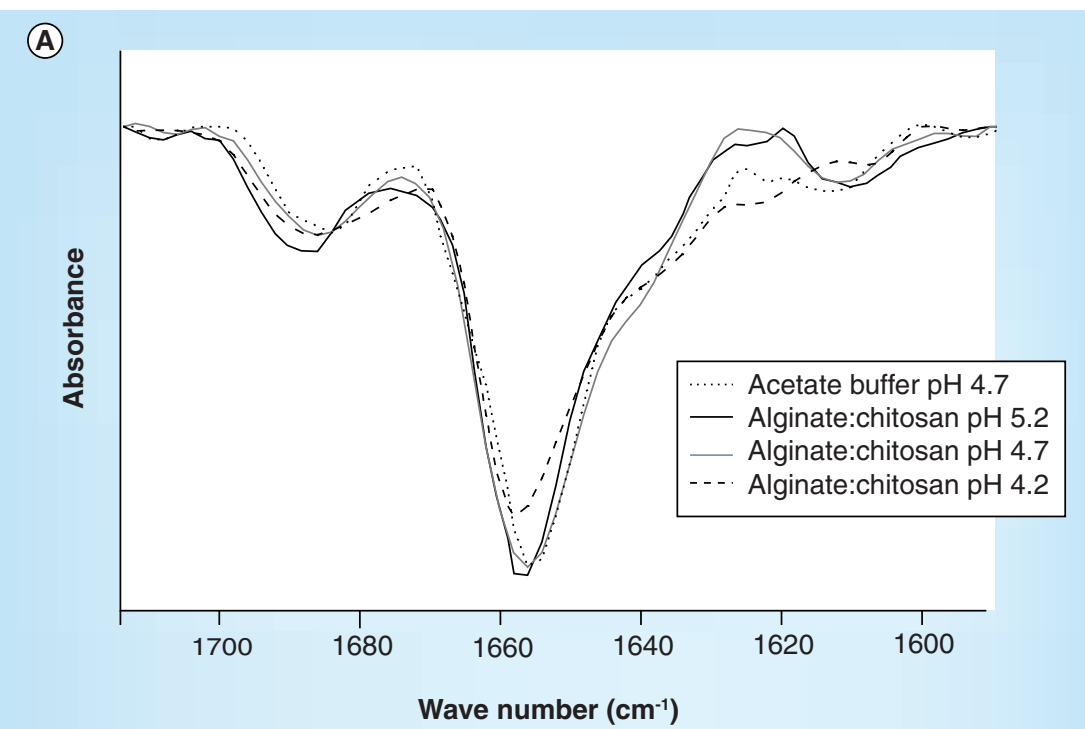

(B)

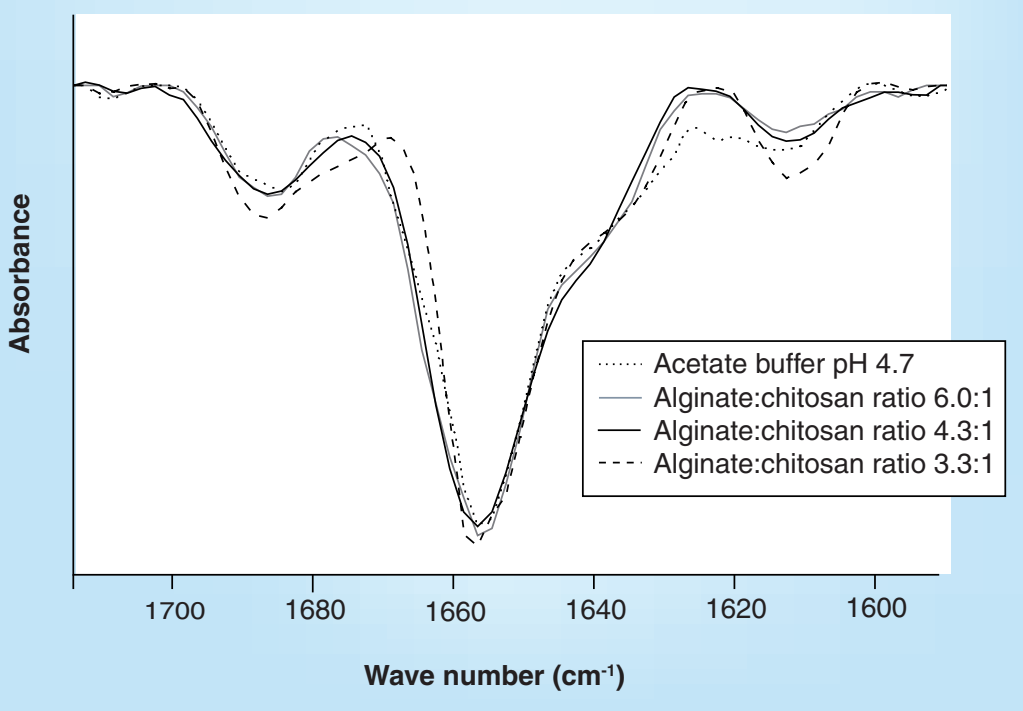

Figure 1. Fourier-transform infrared assessment for structural characterization of insulin encapsulated into nanoparticles.

(A) Second-derivative Fourier-transform infared spectrum of human insulin in solution, $10 \mathrm{mg} / \mathrm{ml}$, acetate buffer $\mathrm{pH} 4.7$ and entrapped into nanoparticles produced with alginate:chitosan mass ratio of 4.3:1 prepared at $\mathrm{pH} 5.2,4.7$ and 4.2. (B) Second-derivative Fourier-transform infared spectrum of human insulin in solution at $\mathrm{pH} 4.7$ and entrapped into nanoparticles produced at $\mathrm{pH} 4.7$ with alginate:chitosan mass ratio of 6.0:1, 4.3:1 and 3.3:1.

Reproduced with permission from [3] (c) Elsevier. 


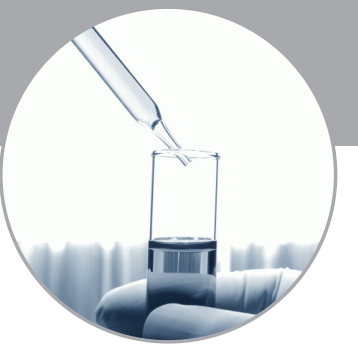

correlates with the in vivo absorption of proteins encapsulated into nanoparticles [13]. Our studies have been focused on the relationship between in vivo oral absorption of proteins and the apparent permeability coefficients obtained in vitro on cell co-cultures and later on isolated intestinal tissue. A new intestinal cell model is being established by us regarding the higher protein absorption rate, at the same time maintaining the biological activity of the studied proteins. The mechanism of protein absorption must be clearly highlighted through different methodologies as well as the relationship between the protein carrier and the efficacy of absorption.

The main advantage of in vivo models is the integration of the dynamic components of the mesenteric blood circulation, the mucous layer and all the other factors that can influence absorption. The disadvantage of in vivo models is that it is impossible to separate the variables involved in the process of absorption, not allowing the identification of individual rate-limiting factors.

The current social and scientific concerns about animal welfare, the need for rapid and consistent bioavailability models by pharmaceutical companies and the demands for economi$\mathrm{cal}$ and quick methodologies give us reason for developing in vitro models with a suitable correlation between results of biopharmaceutical absorption in vivo and in vitro.

\section{Polymeric nanoparticles, chitosan \& beyond}

Polymer-based delivery systems have been proposed as valid approaches to provide successful systems to formulate proteins and we have a solid knowledge of the role of polymeric systems and their interaction with protein drugs [14]. In particular, chitosan-based delivery systems have been proposed to provide favorable and protective conditions to deliver biopharmaceuticals. This natural biopolymer has been used in our research to tailor formulations according the biopharmaceutical need. Chitosan exhibits several positive biological properties, such as biocompatibility, biodegradability, nontoxicity and mucoadhesiveness, which make it a promising candidate for the formulation of protein drugs. Chitosan has been successfully applied for protein delivery applications in some of our recent publications due to its targeting ability and oral administration $[15,16]$. We are interested in studying the absorptionenhancement effect of chitosan in intestinal insulin absorption, using chitosan as a polymeric matrix or a nanoparticle coating [17].
Chitosan is also currently being explored by us as an siRNA vector due to its advantageous low toxicity, mucoadhesion, biodegradability and biocompatibility [18]. The system must be small enough to allow internalization into cells and be capable of avoiding endosome-lysosome processing following endocytosis. In physiological fluids, chitosan can effectively bind siRNA and protect it from nuclease degradation. Its cationic polyelectrolyte nature provides strong electrostatic interaction with negatively charged mucosal surfaces and other molecules like siRNA. Consequently, effective siRNA delivery after pulmonary administration is able to release siRNA easily, resulting in sequencespecific post-transcriptional downregulation of target genes [19].

We are presently working on the conjugation of siRNA to chitosan, which may enhance its physicochemical properties, improving efficacy and decreasing toxicity. The cellular specificity is promoted by the linkage of this conjugate to specific ligand moieties targeting surface receptors of cancer cells [20]. When the carrier is engrafted with particular ligands targeting such receptors, it can be employed for specific recognition and interaction with cancer cells. The novelty of this approach is expected to result in clinical improvements for cancer therapy.

\section{Future perspective}

Biopharmaceuticals that will be developed as nanoparticle products should possess a true therapeutic advantage. However, each biopharmaceutical requires a tailor-made manufacturing process and formulation must be designed to meet general requirements, such as the pharmaceutical aspects as well as aspects related to efficacy, safety, patient compliance and healthcare costs.

Our efforts in our projects are intended to result in a profitable product or methodology, able to provide clear benefits aligned with actual therapeutic regimens or to allow the characterization of biopharmaceutical nanoformulations on an in vitrolin vivo correlation basis.

The technology to develop and manufacture biopharmaceuticals as nanoparticles is expected to be available in the near future, although their application may require more time. Toxicological and long-term administration side effects have not yet been fully established, but we believe that the risk-benefit ratio will boost the market, with more and more nanomedicines containing biopharmaceuticals as active drugs. 


\section{Financial disclosure/acknowledgements}

The author would like to acknowledge financial support from Fundação para a Ciência e Tecnologia. The author has no other relevant affliations or financial involvement with any organization or entity with a financial interest in or financial conflict with the subject matter or materials discussed in the manuscript apart from those disclosed. No writing assistance was utilized in the production of this manuscript.

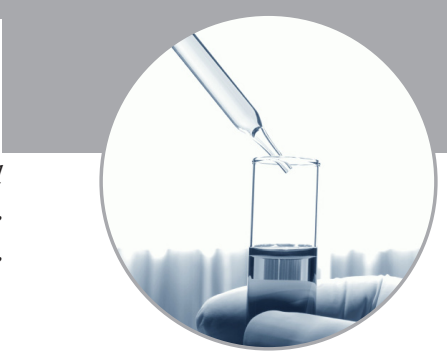

\section{Executive summary}

- Recent years have seen the rise of biopharmaceutical medicines as promising tools for modern therapeutics.

- This new class of therapeutics is quite heterogenous and includes different molecules such as proteins, peptides and nucleic acids, among others, with use in virtually all therapeutic fields and diagnostics.

- Challenges in developing adequate systems that allow the use of biopharmaceuticals in daily life are huge due to their inherent instability, poor pharmacokinetics and potential toxicity.

- Polymeric nanoformulations are being proposed as valid carriers to deal with protein and siRNA stability during manufacturing, shelf-life and administration.

- Tools to assess and control stability of biopharmaceuticals are fundamental to the product development process.

- In vitro biological tools that simulate physiological barriers as close as possible are of increasing interest in drug and formulation screening, as well as understanding the absorption mechanisms of biopharmaceuticals.

\section{Bibliography}

1 Kirchhoff F, Silencing HIV-1 in vivo. Cell 134, 566-568 (2008).

2 van de Weert M, Jorgensen L, Horn Moeller E, Frokjaer S. Factors of importance for a successful delivery system for proteins. Exp. Opin. Drug. Deliv. 2, 1029-1037 (2005).

3 Sarmento B, Ferreira DC, Jorgensen L, van de Weert M. Probing insulin's secondary structure after entrapment into alginate/ chitosan nanoparticles. Eur. J. Pharm. Biopharm. 65, 10-17 (2007).

4 Tatford OC, Gomme PT, Bertolini J. Analytical techniques for the evaluation of liquid protein therapeutics. Biotechnol. Appl. Biochem. 40, 67-81 (2004).

5 Sarmento B, Ribeiro A, Veiga F, Ferreira D. Development and validation of a rapid reversed-phase HPLC method for the determination of insulin from nanoparticulate systems. Biomed. Chromatogr. 20, 898-903 (2006).

6 des Rieux A, Ragnarsson EG, Gullberg E, Preat V, Schneider YJ, Artursson P. Transport of nanoparticles across an in vitro model of the human intestinal follicle associated epithelium. Eur. J. Pharm. Sci. 25, 455-465 (2005).

7 Ziv E, Bendayan M. Intestinal absorption of peptides through the enterocytes. Microcosp Res Techniq, 49, 346-352 (2000).

8 Salamat-Miller N, Johnston TP. Current strategies used to enhance the paracellular transport of therapeutic polypeptides across the intestinal epithelium. Int. J. Pharm. 294, 201-216 (2005).
9 des Rieux A, Fievez V, Garinot M, Schneider YJ, Preat V. Nanoparticles as potential oral delivery systems of proteins and vaccines: a mechanistic approach. J. Control. Rel. 116, 1-27 (2006).

10 Hilgendorf C, Spahn-Langguth H, Regardh CG, Lipka E, Amidon GL, Langguth P. Caco-2 versus Caco-2/HT29MTX co-cultured cell lines: permeabilities via diffusion, inside- and outside-directed carrier-mediated transport. J. Pharm. Sci. 89, 63-75 (2000).

11 Walter E, Janich S, Roessler BJ, Hilfinger JM, Amidon GL. HT29-MTX/Caco-2 co-cultures as an in vitro model for the intestinal epithelium: in vitro-in vivo correlation with permeability data from rats and humans. J. Pharm. Sci. 85, 1070-1076 (1996).

$12 \operatorname{des}$ Rieux A, Fievez V, Theate I, Mast J, Preat V, Schneider YJ. An improved in vitro model of human intestinal follicle-associated epithelium to study nanoparticle transport by M cells. Eur. J. Pharm. Sci. 30, 380-391 (2007).

13 Antunes F, Andrade F, Ferreira D, Sarmento B. Comparison of two triple co-culture in vitro cell models to predict intestinal absorption of peptidic drugs. Presented at: The International Pharmaceutical Federation - FIP - World Congress 2010. New Orleans, LA, USA, 14-18 November 2010.

14 Sarmento B, Ferreira D, Vasconcelos T. Polymer-based delivery systems for oral delivery of peptides and proteins. In: Delivery Technologies for Biopharmaceuticals: Peptides, Proteins, Nucleic Acids and Vaccines. Jorgensen L, Nielson HM (Eds). John Wiley \& Sons (2009).
15 Sarmento B, Ribeiro A, Veiga F, Sampaio P, Neufeld R, Ferreira D. Alginate/chitosan nanoparticles are effective for oral insulin delivery. Pharm. Res. 24, 2198-2206 (2007).

16 Sarmento B, Ribeiro A, Veiga F, Ferreira D, Neufeld R. Oral bioavailability of insulin contained in polysaccharide nanoparticles. Biomacromolecules 8, 3054-3060 (2007).

17 Teixeira J, Gehm C, Júlio A et al. Chitosan as mucoadhesive agent to enhance absorption of therapeutic proteins encapsulated into solid lipid nanoparticles. Presented at: Nanotech Conference and Expo 2009. Houston, TX, USA, 3-7 May 2009.

18 Dass CR, Choong PF. The use of chitosan formulations in cancer therapy. J. Microencapsul. 25, 275-279 (2008).

19 Lee DW, Yun KS, Ban HS, Choe W, Lee SK, Lee KY. Preparation and characterization of chitosan/polyguluronate nanoparticles for siRNA delivery. J. Control Release 139, 146-152 (2009).

20 Correia D, Santana H, Vales A et al. Chitosan-cetuximab conjugate for pulmonary paclitaxel delivery-preliminary concepts. Presented at: 8th International Symposium on Polymer Therapeutics: From Laboratory to Clinical Practice. Valência, Spain, 19-21 May 2010. 